\title{
Crohn's Disease and Urinary Manifestations: A Crohn's Intestinal Disease Mimicking a Bladder Mass
}

\author{
Bronislava Papadatou', Lidia Monti², Giuliano Torre'1, Fabio Panetta1, \\ Antonella Diamanti ${ }^{*}$ \\ ${ }^{1}$ Gastroenterology, Hepathology and Nutrition Unit, "Bambino Gesù” Children's Hospital, Rome, Italy \\ 2Department of Radiology, "Bambino Gesù” Children's Hospital, Rome, Italy \\ Email: antonella.diamanti@opbg.net
}

Received 16 April 2014; revised 30 May 2014; accepted 14 June 2014

Copyright (C) 2014 by authors and Scientific Research Publishing Inc.

This work is licensed under the Creative Commons Attribution International License (CC BY).

http://creativecommons.org/licenses/by/4.0/

(c) (i) Open Access

\begin{abstract}
We report a rare mode of presentation of Crohn's Disease (CD) in a 17-year-old boy, who was admitted at our hospital due to abdominal pain, dysuria, polyuria and fever. The ultrasound showed an increased wall thickness of the small bowel and cecum and a vegetating lesion adjoined into the bladder. The histology was not suggestive of CD and it excluded the bladder neoplasm. Based on the biochemical assessment and the imaging, we started the treatment specific for CD (nutritional therapy by Modulen, short courses of steroids, azathioprine and mesalazine during the follow up), These treatments improved the gastrointestinal symptoms and the nutritional status of our patient but also induced the disappearance of the mass adjoined into the bladder. In spite of the severe onset, in this patient, the clinical course of $C D$ was mild and responder to conventional therapy.
\end{abstract}

\section{Keywords}

Crohn's Disease, Bladder Mass, Adolescents

\section{Introduction}

Crohn's Disease (CD) is a chronic, relapsing and remitting inflammatory disease of the gastrointestinal tract. It has a prevalence of 140 cases per 100,000 in the West [1], and is characterized by transmural, patchy and granulomatous inflammation [1] [2]. The disease can affect any part of the gastrointestinal tract. However,

Corresponding author.

How to cite this paper: Papadatou, B., Monti, L., Torre, G., Panetta, F. and Diamanti, A. (2014) Crohn's Disease and Urinary Manifestations: A Crohn's Intestinal Disease Mimicking a Bladder Mass. Health, 6, 1482-1486. 
ileo-colonic (right lower quadrant) disease is most commonly seen [1] [2]. Anorectal involvement is seen in upto $38 \%$ of patients [3]. The etiology of CD remains unclear, but there is increasing evidence that genetic as well as environmental factors play a role in causing a sustained activation of mucosal immune responses [1] [2]. The incidence of CD seems to be bimodal [1], the first peak occurring in the late adolescence and in the early adulthood. The prevalence of CD in many developed countries is estimated at $0.1 \%$ [1]. Common presenting symptoms include diarrhea, abdominal pain, weight loss, and fatigue [1] [2]. Disorders of the urinary tract are well known complications of Crohn's disease (CD), especially if the terminal ileum is involved [3]. We describe a case where a 17-year-old boy who presented as an emergency with abdominal pain, dysuria, polyuria and fever due to the presence of an urinary bladder mass, revealed a previously undiagnosed CD.

\section{Case Report}

A17-year-old boy was admitted due to abdominal pain, polyuria, dysuria and fever. On admission the weight was $55 \mathrm{Kg}$, height $169 \mathrm{~cm}$, pulse 72/bpm, blood pressure $128 / 78 \mathrm{mmHg}$, and temperature $37.2^{\circ} \mathrm{C}$. On examination, the abdomen was soft, slightly distended, and non-tender; there were no organomegaly or decreased bowel sounds. Rectal examination showed a normal aspect of the region with normal sphincter.

The biochemical detections showedhaemoglobin, leukocytes, platelets, total protein and albumin, glucose, urea and creatinine within the refences values but increased C-reactive protein (CRP)and erythrocyte sedimentation rate (ESR).

Stool cultures and stool tests for Clostridium difficile toxins A and B were negative. Urinalysis was normal and in particular it did not reveal microscopic hematuria.

The trans-abdominal ultrasound found increased wall thickness of the small bowel and of the cecum but also a vegetating lesion adjoined in the bladder with a diameter of $1 \mathrm{~cm}$ (see Figure 1(a) and Figure 1(b)). Magnetic Resonance Imaging (MRI) confirmed the presence of a mass adjoined into the bladder and it also revealedmesentericfat tissue hypetrophy, dysomogeneous hyperenhancement of the thickened bowel wall anddilatation of the terminalileum (see Figures 2(a) and Figure 2(b)).

It was supposed that the last loop bowel wall thickening could have involved also the wall of the bladdermimicking mass adjoining into the lumen. Upper and lower gastrointestinal endoscopy and a bladder cystoscopy in the same setting were performed, to better detect the nature of the bladder mass and to exclude potential comorbidity for both IBD and bladder neoplasia. In the meanwhile, due to the suspected IBD, perinuclear antineutrophil cytoplasmic antibody (pANCA), anti-saccharomyces cerevisiae antibody (ASCA) and fecal calprotectin (FC) were carried out, with positive results for ASCA (IgA 156.2, IgG 77 Abs. C/P, RV < 25 IU/ml respectively) and FC $(952 \mu \mathrm{g} / \mathrm{g}, \mathrm{RV}<100 \mu \mathrm{g} / \mathrm{g})$ but negative ANCA.

The upper endoscopy showed mild rigidity of the pylorus; the colonoscopy revealed stenosis of the intestinal lumen in the cecal region with presence of pseudopolyps and ulcerations. Histopathology was suggestive of anacutecolonic inflammation and a chronic gastritis, without features suggesting CD.

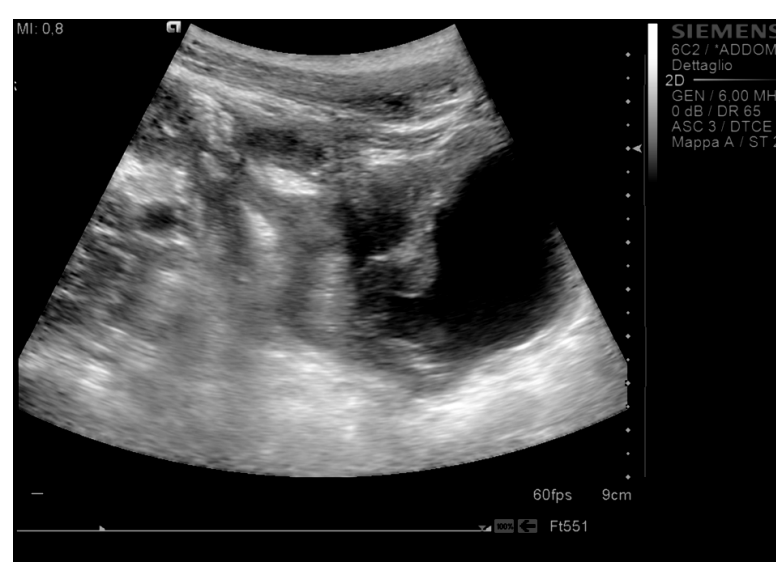

(a)

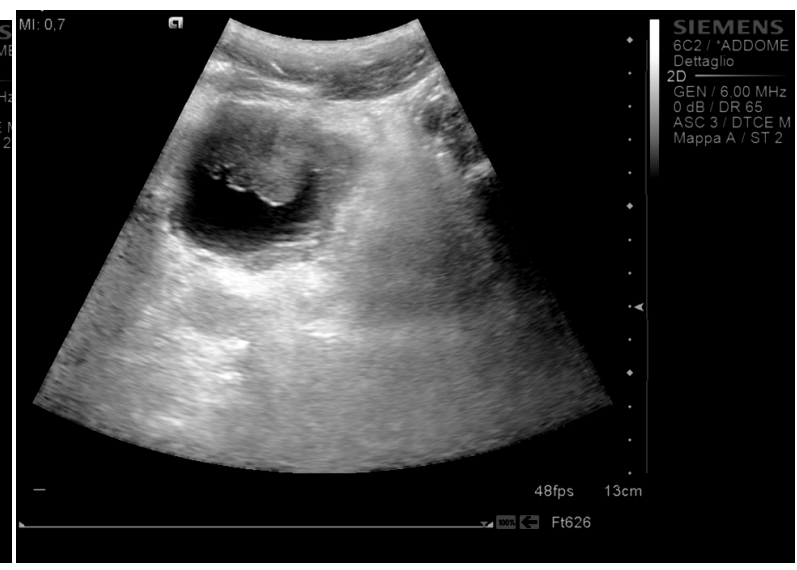

(b)

Figure 1. (a) Ultrasound trasversal scan: Vegetating lesion in the bladder; (b) Ultrasound longitudinal scan: Vegetating lesion in the bladder and last loop with bowel wall thickening. 


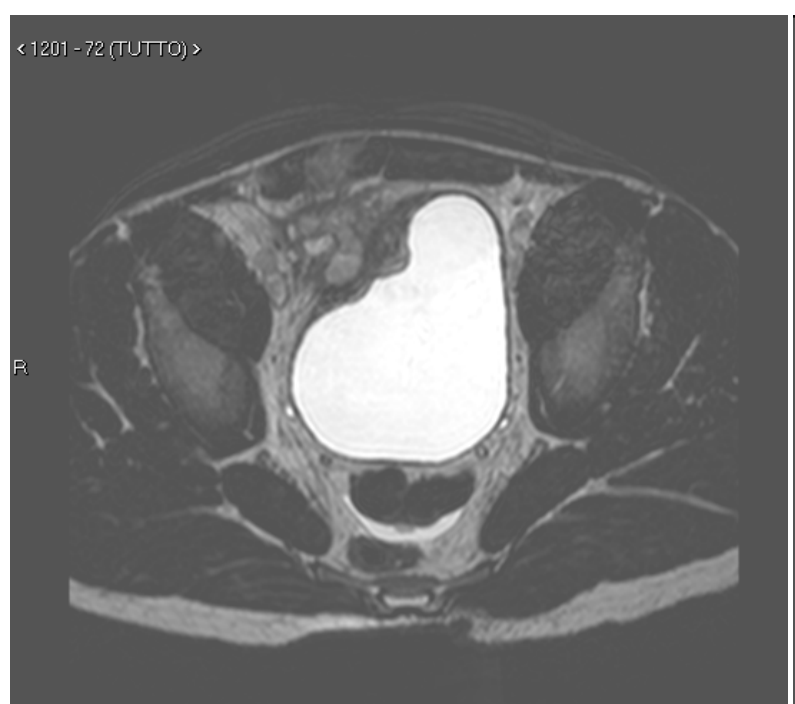

(a)

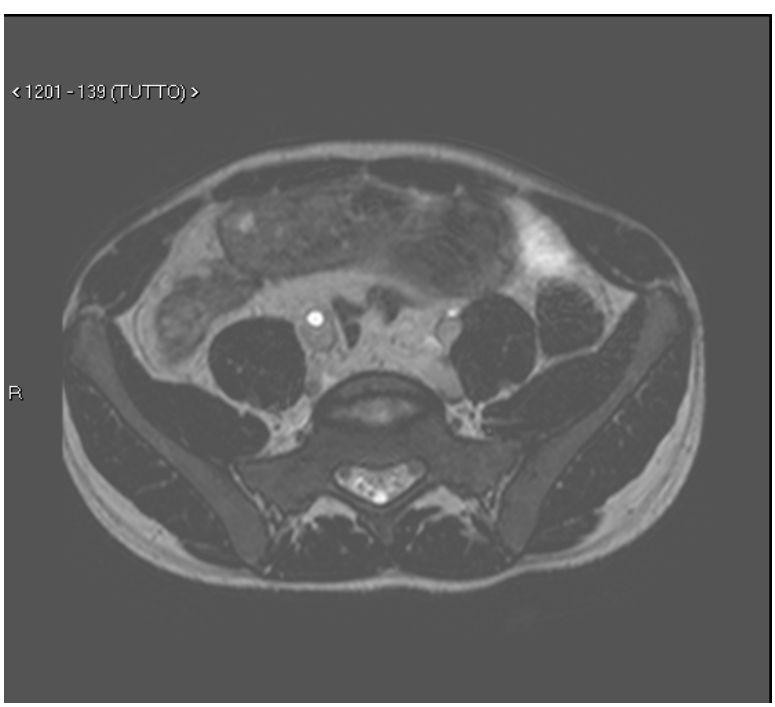

(b)

Figure 2. Ultrasound trasversal scan: Normal bladder, regression of the vegetating lesion.

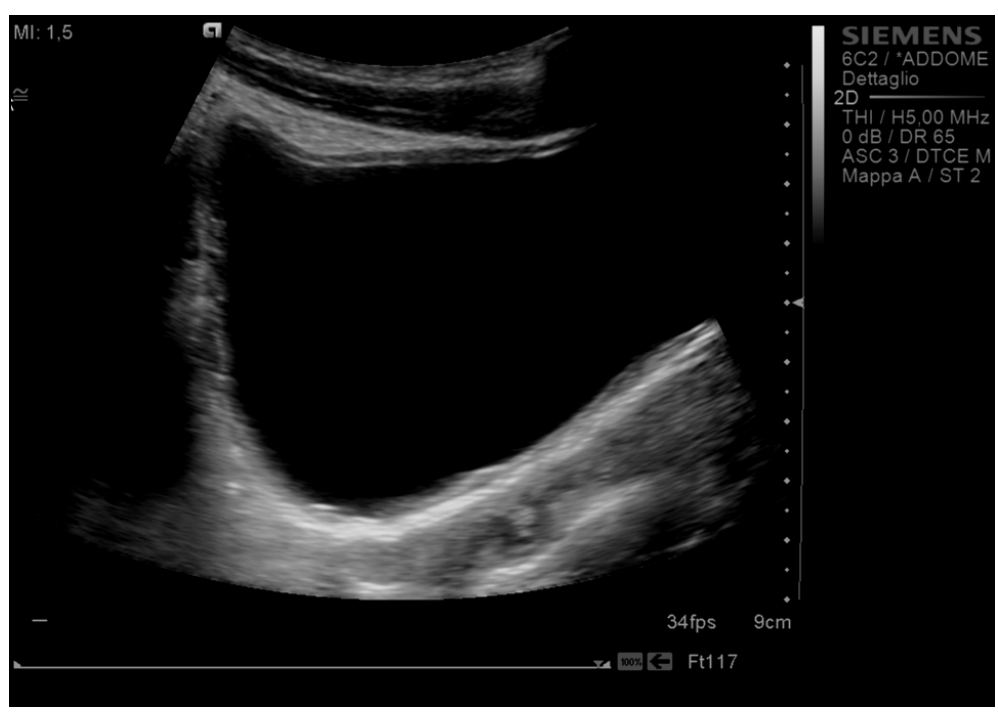

Figure 3. Ultrasound trasversalscan: Normal bladder, regression of the vegetating lesion.

The cystoscopy showed in the area of the dome of the bladder a superficial, large and sessilepapillary lesion, with a diameter of $1 \mathrm{~cm}$. Histology specimens revealed a normal aspect of the urothelialepithelium. Therefore the lesion described was considered as an extroflession of the bladder mucosa, due to the compression exerted by the bowel.

Although histology had not been clearly suggestive of CD, the clinical symptoms, the laboratory evaluations and imaging led us to approach a pharmacologic treatment specific for CD. Thus we started the nutritional therapy with exclusive Modulen (2.5 L/day) combined with mesalazine, ciprofloxacin and metronidazole. A furthe rlower endoscopy with ballon dilatation of the cecum was performed after two weeks from the previous. The dilatation of the cecum allowed to detect the ileo-cecal valve and the last loop of the ileum. On the ileo-cecal valve ulcers and pseudopolyps were observed, while in the last bowel loop the mucosa appeared normal except for the presence of a small ulceration next to the ileo-cecal valve. The histologic evaluation of the specimens demonstrated the presence of lymphoidaggregatesin both the colon and the cecum combined with cryptic abscesses and neutrophil infiltrates more suggestive of an inflammatory bowel disease than the first one. Therefore we contin- 
ued thetreatment with Modulen and mesalazine. During the follow-up, our patient required short courses of steroids for the relapse of abdominal pain and diarrhea. Thus azathioprine was added and a small amount (1 litre/day) of Modulen was continued as supplementation to a free diet.

After 2.5 years from the diagnosis, our patient shows normal biochemical assessment, including CRP and ESR. The ultrasound examination of the bladder shows a complete regression of the vegetating mass (see Figure 3). The patient is doing well, his weight is $65 \mathrm{Kg}$ and his height is $170 \mathrm{~cm}$; the CDAI index is 0 . He assumes mesalazine, azathioprine (1.5 mg/kg/day) and Modulen (1 litre/day).

\section{Discussion}

The topic of urogenital complications of CD is justly narrow but clinically relevant [3]. The prevalence of urologic complications in CD seems to be variable, ranging from 4\% to 23\% [3]. CD patients with urinary complications may present with urinary complaints prior to gastrointestinal abnormalities [3] [4]. Furthermore, urinary symptoms, as our case outlines, may reveal the CD presentation, before the urinary complications have occurred. In our case, indeed, the urinary symptoms, although associated with a clinical history positive for abdominal pain, revealed a picture of CD. The clinical course of the CD in this patient, in spite of the severe onset, has been mild until now. The nutritional therapy with Modulen as exclusive diet and as supplementation, combined with short courses of steroids and azathioprine, maintained indeed the patient on clinical remission, avoiding that the bladder involvement worsened up to the fistulization. The early recognition of the CD may indeed impede that the fistulizing process develops, due to the direct extension of the inflammatory process from a continuous loop of the bowel [5].

Common and mild urinary CD-related complications include infections and urolithias is caused by the defect of oxalate metabolism [3] [4]. Rare although serious occurrences, associated with penetrating-type CD, include stricture of the right ureter due to peri-ileal inflammation and entero-urinary fistulization [3] [4] [6]. Fistula formation between the bladder and some parts of the ileo-colonic tract is a severe complication of CD, which often requires surgical intervention [3] [4]. Clinically, urinary complaints such as recurrent infections, fever, dysuria, pneumaturia, and fecaluria should be thoroughly investigated and clearly stated on radiology requests, to avoid missed or delayed diagnoses [3] [4] [6]. Two cases of proven metastatic granulomatous lesion of the urinary bladder have been also reported [7]. A few cases of bladder cancer, have been also described during the course of the CD, but not in young patients [8] [9]. Bladder carcinoma is predominant during the fifth, sixth, and seventh decades and the exact cause is unknown; the protracted use of immunosuppressive drugs and steroids, with consequently decreased immune function, nevertheless, has been implicated [6].

In conclusion, we have reported a rare mode of presentation of CD in an adolescent. The clinical symptoms and the imaging suggested an urinary disease. However, the normal aspect of the bladder specimens, the biochemical assessment and the imaging suggestive of CD, allowed us to start the treatment for CD, that improved the gastrointestinal symptoms but also induced the disappearance of the mass into the bladder. Interestingly, this is the second report of an adolescent with this novel presentation. Recently there has been a case strictly related to an adolescent boy, who had a complete clinical resolution with conventional therapy [9]. In both cases, in spite of the severe onset, the clinical course was mild, thus suggesting that in adolescent patients with CD the clinical course may be unrelated with the mode of presentation.

\section{Sources of Funding}

Sponsors had no such involvement.

\section{Conflict of Interest Statement}

There is no conflict of interest that authors should disclose.

\section{References}

[1] Loftus Jr., E.V. (2004) Clinical Epidemiology of Inflammatory Bowel Disease: Incidence, Prevalence, and Environmental Influences. Gastroenterology, 126, 1504-1517. http://dx.doi.org/10.1053/j.gastro.2004.01.063

[2] Cummings, J.R., Keshav, S. and Travis, S.P. (2008) Medical Management of Crohn’s Disease. BMJ, 336, $1062-1066$. http://dx.doi.org/10.1136/bmj.39547.603218.AE 
[3] Pardi, D.S., Tremaine, W.J., Sandborn, W.J., et al. (1998) Renal and Urologic Complications of Inflammatory Bowel Disease. The American Journal of Gastroenterology, 93, 504-514. http://dx.doi.org/10.1111/j.1572-0241.1998.156_b.x

[4] Joffe, N. (1976) Roentgenologic Abnormalities of the Urinary Bladder Secondary to Crohn's Disease. American Journal of Roentgenology, 127, 297-302. http://dx.doi.org/10.2214/ajr.127.2.297

[5] Merine, D., Fishman, E.K. and Huhlman, J.E. (1989) Bladder Involvement in Crohn's Disease: Role of CT in Detection and Evaluation. Journal of Computer Assisted Tomography, 13, 90-93. http://dx.doi.org/10.1097/00004728-198901000-00019

[6] Molnár, T., Tiszlavicz, L., Balogh, D.A., et al. (2000) Crohn’s Disease of the Bladder. A New Type of Metastatic Granulomatous Inflammatory Disease? The American Journal of Gastroenterology, 95, 850-851.

[7] Fujimura, S.K.Y., Kihara, T., Uchida, J., et al. (1992) Transitional Cell Carcinoma of the Bladder Associated with Crohn's Disease: Case Report and Review of the Literature. The British Journal of Radiology, 65, 1040-1042. http://dx.doi.org/10.1259/0007-1285-65-779-1040

[8] Juan, C., Patron, U.M., Khurrum, B.J.J., et al. (2003) An Unusual Way to Diagnose a Rare Case of Bladder Carcinoma in a Patient with Crohn's Disease. International Journal of Colorectal Disease, 18, 172-173.

[9] Evans, C.E. and Tighe, M.P. (2014) Crohn’s Disease Presenting as Bladder Mass. Archives of Disease in Childhood, 99, 256. http://dx.doi.org/10.1136/archdischild-2013-305500 
Scientific Research Publishing (SCIRP) is one of the largest Open Access journal publishers. It is currently publishing more than 200 open access, online, peer-reviewed journals covering a wide range of academic disciplines. SCIRP serves the worldwide academic communities and contributes to the progress and application of science with its publication.

Other selected journals from SCIRP are listed as below. Submit your manuscript to us via either submit@scirp.org or Online Submission Portal.
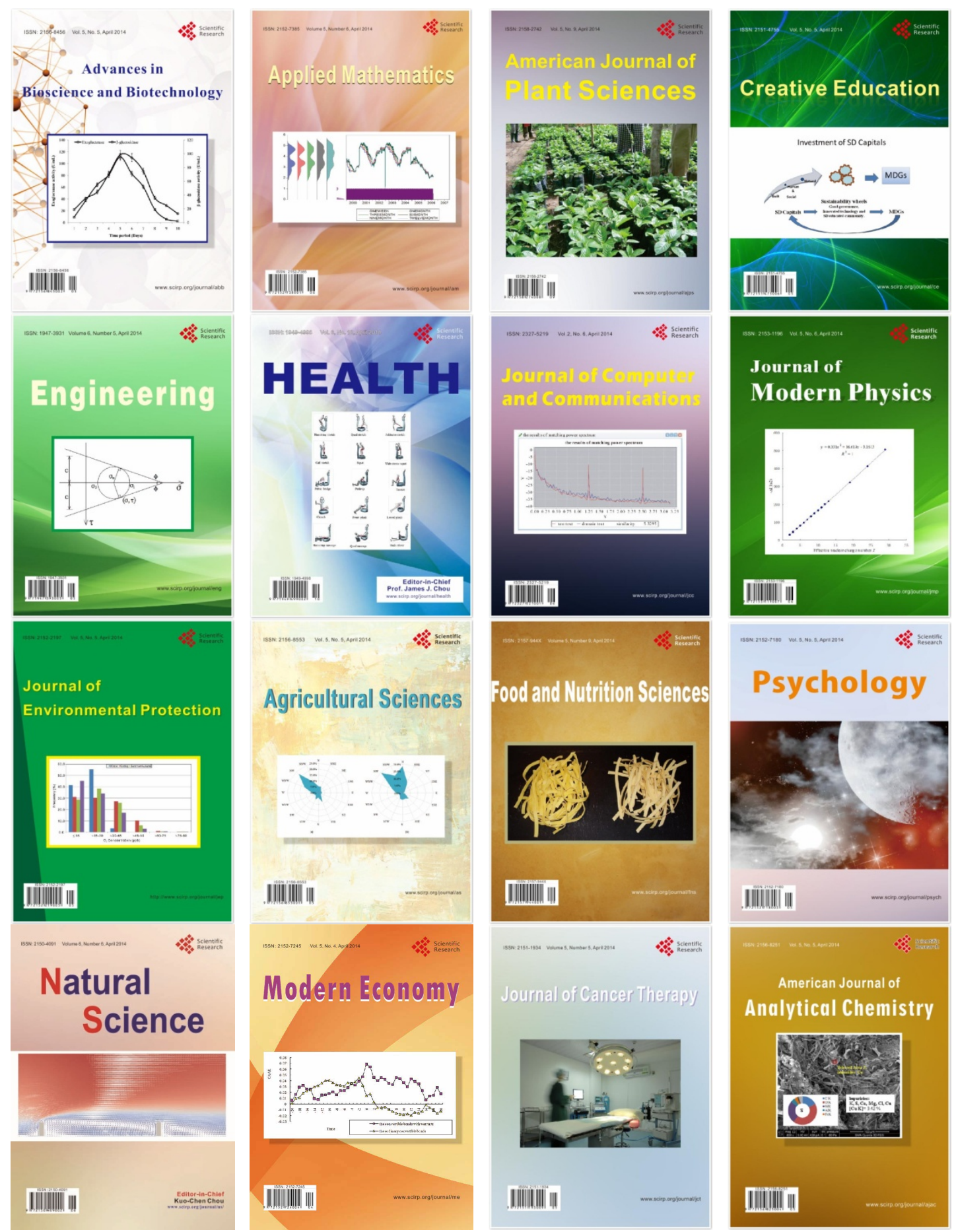